Research Article

\title{
Latent Variable Modeling Within an Ecosystems Approach: ICT Use in Schools
}

\author{
Z. Georganta ${ }^{1,2 *}$ and K. Keramida ${ }^{3}$ \\ ${ }^{I}$ University of Macedonia, Thessaloniki, Dept. of Economics and Social Sciences, Greece. \\ ${ }^{2}$ Member of the Hellenic Statistics Authority (EL.STAT.), Pireas, Attiki, Greece. \\ ${ }^{3}$ Secondary Education Institute of N. Ionia, Thessaloniki, Greece.
}

\begin{abstract}
Socio-economic phenomena are usually measured with error which, more often than not, is of a systematic nature rather than random, as traditional econometric models assume. Thus, regression analysis may come to incorrect conclusions with disastrous socio-economic consequences resulting from erroneous policies which are designed on inaccurate econometric estimates. Latent variable modeling (LVM) has been used to resolve this problem by considering the explicit specification of a non-random error. This paper presents an LVM explanation of why Information and Communication Technologies (ICTs) are not used more in schools. The LV model is specified by using a metaphor from a physical phenomenon, thus showing the usefulness of combining approaches from different scientific fields.
\end{abstract}

Keywords: Latent variable modeling, Information and communication technologies, eco-systemic approach.

\section{Introduction}

LVM has been used in social sciences and economics to resolve successfully the problem of statistical and econometric analysis of phenomena which cannot be accurately expressed in a quantitative dimension [1-3]. More frequently than not, socio-economic data contain measurement errors which cannot be considered as random. This is a serious problem if we intend to use traditional regression analysis because one of its basic assumptions is the randomness of the error term. If the error term is systematic and not random, then we are lead to distorted conclusions. Taking into account the fact that in social sciences econometric estimates are used by decision makers to design socio-economic policies, it is obvious that countries can end up in disasters.

Social sciences try to explain human behavior which is characterized by highly probabilistic features. Thus, a lot of social and economic data are expressed as indicators calculated on the basis of a Likert-scale type of answers within the framework of a statistical questionnaire survey. Such data are used to compile the so called observed indicator variables or factors, which are considered as approximations of the underlined theoretical concepts, which are the so called latent variables. LVM uses the analysis of variance-covariance to study the complex path structure of direct and indirect interdependencies of observed factors and their influence on the latent phenomena under investigation. The LVM approach is based on the following three-fold postulation:

\footnotetext{
*E-mail address: zoe@vom.gr; z.georganta@statistics.gr ISSN: 1791-2377@ 2011 Kavala Institute of Technology. All rights reserved.
}

(1) Formulation of the hypothesis to be investigated as a causal structure among a set of latent or theoretical variables.

(2) Detection of a set of observed factor-variables, which can be used as proxies of the latent variables. Such observed variables are called indicator variables.

(3) Specification of the latent variables as functional combinations of the indicator variables and measurement errors in a causal chain of observed and non-observed variables.

The aim of this paper is two-fold: first, the main points of the LVM econometric methodology will be presented, and second, the LVM methodology will be applied to explain the slow adoption of ICTs in class, taking as a case study the Mathematics course in the secondary education in the city of Thessaloniki, Greece. For this empirical exercise we have adopted an eco-systemic approach to help us come up with the model specification. The paper is structured in four sections: the next section gives a concise description of the LVM approach to econometric analysis. The third section describes the empirical exercise and the final section concludes the paper.

\section{The LVM Approach}

The general form of a simultaneous latent variable model includes three equations which are described as follows:

$\underset{(\mathrm{mx1})}{\eta}=\underset{(\mathrm{mxm})}{\mathrm{B}} \underset{(\mathrm{mxl})}{\eta}+\underset{(\mathrm{mxn})}{\Gamma} \underset{(\mathrm{nx} 1)}{\xi}+\underset{(\mathrm{mx1})}{\zeta}$ 
Structural Model

$\underset{(\mathrm{px} 1)}{\mathrm{y}}=\underset{(\mathrm{pxm})}{\Lambda_{\mathrm{y}}} \underset{(\mathrm{mx} 1)}{\eta}+\underset{(\mathrm{px} 1)}{\varepsilon}$

Measurement Model for $\mathrm{y}$

$$
\underset{(\mathrm{q} x 1)}{\mathrm{x}}=\underset{(\mathrm{q} \times n)}{\Lambda_{(\mathrm{nx} 1)}} \underset{(\mathrm{qx} 1)}{\xi}
$$

\section{Measurement Model for x}

where $\eta$ and $\xi$ are random vectors of latent dependent and independent variables, respectively, $B$ and $\Gamma$ are coefficient matrices, and $\zeta$ is a random vector of disturbance terms. The elements of B represent direct causal effects of $\eta$-variables on other $\eta$-variables and the elements of $\Gamma$ represent direct causal effects of $\xi$-variables on $\eta$-variables. The vectors $\eta$ and $\xi$ are not observed, but instead vectors $\mathrm{y}$ and $\mathrm{x}$ are observed in such a way that the two measurement models (2) and (3) are valid. $\Lambda_{\mathrm{y}}$ and $\Lambda_{\mathrm{x}}$ are coefficient matrices, and $\varepsilon$ and $\delta$ are vectors of errors of measurement in $\mathrm{y}$ and $\mathrm{x}$, respectively.

The observed vectors $\mathrm{y}$ and $\mathrm{x}$ contain indicator variables for the unobserved or latent variables $\eta$ and $\xi$, respectively. The latent variables correspond to theoretical constructs or variables measured correctly. For this reason, they are called "true" variables. The structural equation model (1) describes the causal relationship between the "true" or latent variables $\eta$ and $\xi$. The measurement models (2) and (3) describe the way in which the latent variables $\eta$ and $\xi$ are measured in terms of the observed variables $\mathrm{y}$ and $\mathrm{x}$, respectively. It is emphasized that $\zeta$ in equation (1) is a vector of classical disturbances, including all random discrepancies that emerge between the actual values of $\eta$ and the values that would be obtained by the corresponding exact or, in the case of no disturbances, stable functional relationship. Such random discrepancies may be due to omitted variables from the model, or to some "intrinsic" randomness in elements of vector $\eta$ which cannot be explained anyway, or to any other non-systematic influence on vector $\eta$ which cannot be captured by the right-hand part of equation (1) no matter how elaborate it is. What $\zeta$ does not include are measurement errors, which are instead incorporated in the vectors $\varepsilon$ and $\delta$ of equations (2) and (3). For a more detailed description of the above model, see among others Joreskog (1977, 1990), Joreskog et al. (1984) and Bolen (1989) [4-7]. Schema 1 gives an example of the path diagram implied by the simultaneous equations (1)-(3). Schema 2 gives an empirical example of a path diagram.

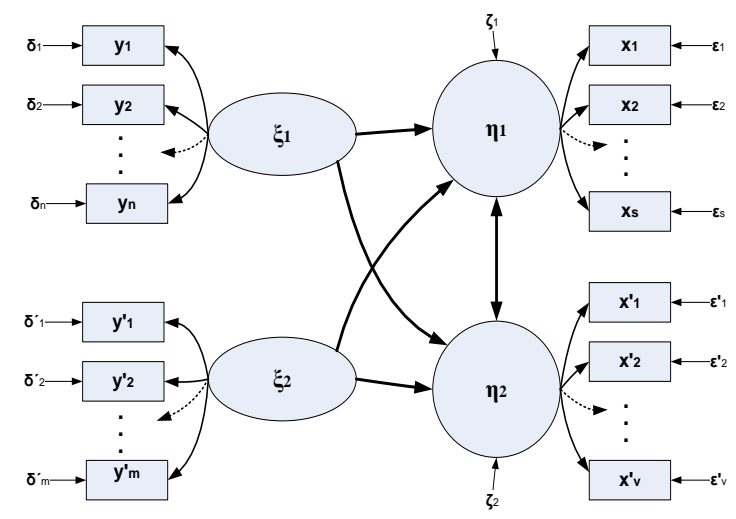

Schema 1: Interrelationships between $\eta 1$ (e.g. product quality), $\eta 2$ (e.g. $\mathrm{R} \& \mathrm{D}$ expenditures), $\xi 1$ (e.g. national institutions), and $\xi 2$ (e.g. firm organization)

\section{Modeling the inadequate use of ICTS in class}

The purpose of this section is to model an answer to the question, "why computers are not used more in class?" The concept of "computer uses in class" represents a complex content for which we can use a metaphor in order to develop insights into it. Zhao and Frank (2003) [8] have described the rapid successful invasion of zebra mussels from the Caspian Sea into the ecosystem of the Great Lakes in Canada-USA border line. Studying this phenomenon it was found out that this was the result of many factors working together and interacting among themselves within the Great Lakes ecosystem. It is noted that ecosystems contain both abiotic and biotic communities.

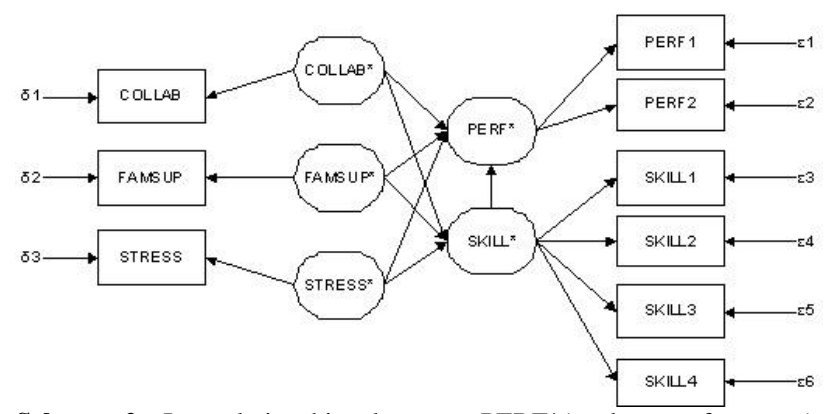

Schema 2: Interrelationships between PERF*(student performance), SKILL*(learning skill acquisition), COLLAB* (students' collaboration), FAMSUP*(family support) and STRESS*(students' stress)

It was, thus, thought that the concept of "school" contains both living and non-living components which interact with each other. So, schools can be considered in terms of systems in the sense of their interconnectivity characteristic. Within this framework, four metaphorical equivalents are established:

(1) schools are ecosystems,

(2) ICT uses are living species,

(3) teachers are members of a keystone species (i.e. species having a controlling influence over the ecosystem), and

(4) external educational innovations are invasions of exotic species.

Within this eco-systemic framework we specified the following path model:

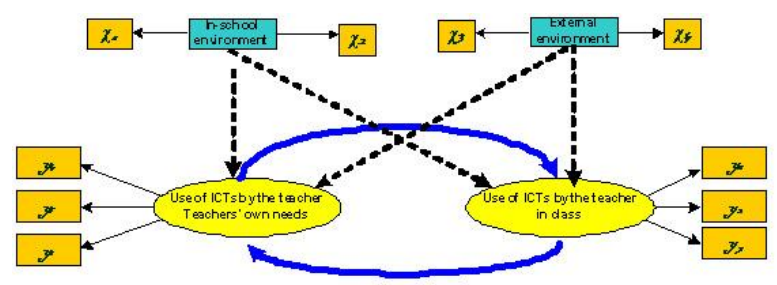

Schema 3. General Model - Interrelationships between ICT use in class, for teachers' own needs and environmental variables

\section{The data and the estimates}

We used data based on (Keramida, 2009) [9]. The Keramida data base includes 606 observations for 26 variables. Our sample for this exercise includes 101 observations drawn randomly from the Keramida data base. The variable notation is describes in Table 1 . It is noted that all variable 
are indicators compiled on the basis of the questionnaire survey in Keramida (2009). Application of the maximum likelihood estimation method for the general model of Schema 3 gives the path model of Schema 4.

Table 1: Variable Notation

\begin{tabular}{lll}
\hline & Notation & Description \\
1 & USE_T & ICT use by the teacher in class \\
2 & USE_NT & ICT use by the teacher for his/her own needs \\
3 & SCH_ENV & School environment to encourage ICT use \\
4 & ATTID & Teacher's attitude towards ICTs \\
5 & TEACH & Use of ICTs in teaching \\
6 & USE_SOFT & Use of software \\
7 & MAN_SOFT & Use of managing software \\
8 & ADM & Use of ICTs in administration \\
9 & PREP & $\begin{array}{l}\text { Use of ICTs for teacher's own preparation to } \\
\text { teach }\end{array}$ \\
10 & OWN_ED & Use of ICTs for teacher's self education \\
\hline
\end{tabular}

The stars represent latent variables. USE_T* and USE_NT* are endogenous latent variables, and SCH_ENV* and ATTID* are exogenous latent variables.

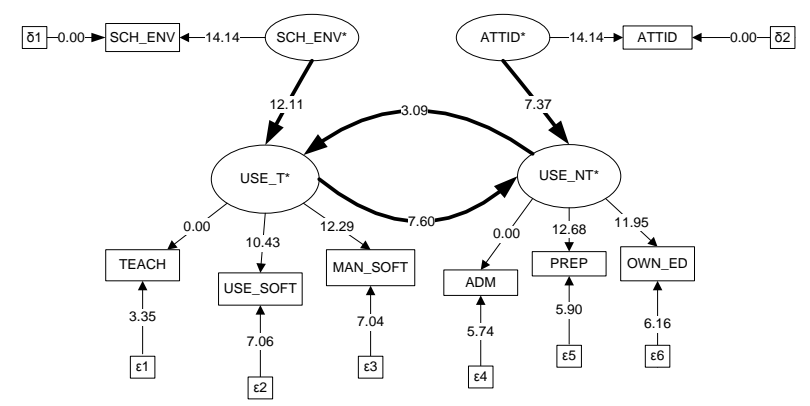

Schema 4: Empirical path model of ICT use in class and for teachers' own needs

The reported numbers in Schema 4 are the t-values at $5 \%$ significance level. We can see that use of ICTs by teachers for their own needs affects significantly their use of ICTs in teaching and vice versa. Also, we can see that there is a positive and significant relationship between a school environment which encourages ICT use, like availability of computers and internet, and the use of ICTs by the teacher in class. Teachers' positive attitude towards computers has a significant effect on teachers' use of ICTs for their own needs in preparing for class, in their self education and in school administration matters. We should also note that measurement errors, both $\varepsilon$ and $\delta$, are significant indicating that the use of a latent variable model is the appropriate one.

\section{Concluding Remarks}

This paper used an LVM approach within an ecosystemic framework in order to study the question of why ICTs are not used more in class. The empirical exercise was based on data from Greece collected by a questionnaire survey covering the Mathematics teachers in secondary education. The results showed that measurement errors were statistically significant implying that a latent variable model is appropriate to study this social phenomenon: the estimated model belongs to the general LVM methodology including the simultaneous equations (1)-(3) which specify explicitly the measurement errors $\varepsilon$ and $\delta$, thus allowing $\zeta$ to include only random components.

Our findings are useful for policy formulation towards increasing the use of ICTs in schools: First, encouraging teachers to make more use of ICTs for their own information and self-education leads them to make more use of ICTs in class. Second, by improving the ICT infrastructure in schools, we end up making greater use of computers in teaching and learning. Third, by using an ecosystems metaphor we were able to develop an LV model to explain an educational phenomenon which would be very difficult to study otherwise, thus showing that combining knowledge from different fields of study may lead to useful conclusions. We can of course apply the experiment with other than Mathematics courses and extend the sample to include the whole of Greece.

\section{References}

1. Georganta Z., "Latent Variable Models in Economics". In T. P. Lianos (ed). Essays in Economic Analysis, Center of Planning and Economic Research - KEPE, 111 (2004).

2. Georganta Z. and Hewitt, W. D., "Information Economy and Educational Opportunities: A Latent Variable Model of Learning Skills". Frontiers in Education. IEEE, Savannah, USA, (2004).

3. Georganta Z. and M. Vogiatzi., "The Effects of ICTs on Ebusiness Performance: Designing a Path Analysis Framework". Journal of Information Technology Impact, Vol.10, Issue 3, (2010).

4. Jöreskog K. G., "Structural Equation Models in the Social Sciences: Specification, Estimation and Testing", in P.R. Krishnaiah (ed.) Applications of Statistics, North Holland, (1977).

5. Jöreskog K. G., "New Developments in LISREL: Analysis of Ordinal Variables using polychoric correlations and weighted least squares", Quality and Quantity, 24, 387, (1990).

6. Jöreskog K. G. and Sörbom D., LISREL VI, Analysis of Linear Structural Relationships by the Method of Maximum Likelihood, User's Guide. Scientific Software: Mooresville, IN, (1984).
7. Bollen K. A., Structural Equations with Latent Variables. John Wiley \& Sons: New York, 1989.

8. Zhao Y. and Frank K.A., "Factors Affecting Technology Uses in Schools: An Ecological Perspective". American Educational Research Journal, Vol.40, No. 4, (2003).

9. Keramida K., ICTs in Teaching Mathematics in Secondary Education: An Ecosystems Approach, Ph.D. Thesis, Department of Applied Informatics, University of Macedonia of Economic and Social Sciences, Thessaloniki, Greece, (2009). 